\title{
The rehabilitation of stroke patients at community health centres in the Western Cape
}

\begin{abstract}
The structure and process of rehabilitation of stroke patients affects the outcomes of the patients. The aim of this study was to determine the structure and process of rehabilitation of stroke patients at Community Health Centres (CHCs) in the Western Cape, South Africa. A quantitative descriptive study was conducted. Questionnaires and archived records were used to collect the data. The study sample used to collect information related to the structure consisted of therapists (16) employed at the centres; while the study sample used to collect information related to the process consisted of 100 first time stroke patients. Descriptive statistics were conducted using Excel and SPSS. The results of the study revealed that there is a lack of occupational and speech therapy services at the centres forming part of the study sample. At centres where these services are provided the frequency and intensity with which the services are received by the patients is extremely low. Further research is needed to determine if the decreased intensity is only as a result of decreased availability of services or if inability of stroke clients to access the services also plays a role.
A Rhoda (MSc),
R Mpofu (PhD), W DeWeerdt (PhD)

KEYWORDS: STROKE, REHABILITATION, COMMUNITY HEALTH CENTRES, STRUCTURE, PROCESS.

\section{INTRODUCTION}

The concept of rehabilitation includes “....all measures aimed at reducing the impact of disability on an individual, enabling him or her to achieve independence, social integration, a better quality of life and self-actualisation" (Helander, 1999). The goals of rehabilitation would be to reduce the impact of the disability on the individual. Although the overall goals of rehabilitation are clear, rehabilitation interventions, providers, context and patient characteristics can differ (Hoenig, Horner, Duncan, Clipp, \& Hamilton, 1999). This makes the implementation of rehabilitation research intricate.

The Structure, Process and Outcome (SPO) framework has been suggested as a method to help reduce this intricacy in research. (Hoenig et al.,1999). This framework which was initially developed to assess the quality of health care has been lately used in rehabilitation research. In this framework, the structure of care includes the facilities available for rehabilitation and the rehabilitation professionals required to provide the treatment. When determining the structure of care information relating to the qualifications of the health professionals providing the service is also investigated. Professionals that are involved in stroke rehabilitation include physiotherapists, occupational therapists, speech therapists, dieticians and nurses. Therapy assistants also assist in the rehabilitation process.

The process of care is defined as the content of care, the referral process to and through the system, as well as the services that the patient receives. Components that are included in the process of care are: provision of assistive devices and prostheses, type of exercises given, use of physical agents such as ultrasound as well as frequency and duration of rehabilitation (Hoenig et al., 1999). Process of care can be evaluated in terms of direct patient care as well as policies and procedures that govern the care provided. Outcomes can be described as patient characteristics that change as a result of care (Hoenig et al., 1999). ${ }^{1}$

Stroke rehabilitation can occur in different settings, which include in-patient

1 Although the outcomes of stroke patients accessing the CHCs were determined, the present manuscript only presents information relating to the structure and process of rehabilitation at the centres.

hospital-based settings, out-patient community-based settings as well as the patients' home. It has been found that stroke patients managed in a stroke unit in the acute stage have better outcomes (Stroke Unit Trialists' Collaboration, 2006). The South African National Guideline on Stroke and Transient Ischaemic Attack Management (2001) proposed that all stroke patients should be admitted to hospital and receive multi-disciplinary rehabilitation. The guideline advocates for a structured approach to the management of stroke patients in which acute management takes place at a hospital and sub acute management is located at specialised rehabilitation centres, secondary inpatient facilities or CHCs. In a study conducted at a $\mathrm{CHC}$ in the Western Cape it was however found that the majority of

\section{Correspondence to:}

\section{A Rhoda}

University of the Western Cape

Private Bag X17,

Bellville

Western Cape Province

South Africa

7535

Email: arhoda@uwc.ac.za 
stroke patients referred to the centre were still in the acute stage post-stroke (Rhoda and Hendry, 2006). Earlier studies conducted elsewhere in South Africa have also highlighted that stroke patients are discharged from in-patient facilities even though community rehabilitation services have been found to be inadequate for the management of acute and sub-acute patients (Fritz, 1995; Hale and Wallner, 1996). The reality of stroke rehabilitation in the South African context is therefore not consist with international trends or with local policy guidelines on [stroke] rehabilitation. Community Health Centres which have been designated as low intensity rehabilitation facilities (Provincial Government of The Western Cape, 2007) often have to manage stroke patients who are in the acute stage (Rhoda, 2002). It becomes important to examine rehabilitation services at $\mathrm{CHCs}$ in the Western Cape more closely in order to obtain a South African perspective regarding stroke rehabilitation.

In order to do this, the present study therefore aimed to determine the Structure and Process of the rehabilitation of stroke patients at $\mathrm{CHCs}$ in the Western Cape, South Africa. When examining the structure of care, the present study attempted to describe the facilities available for rehabilitation and the rehabilitation professionals required to provide treatment. The type, content and intensity of rehabilitative therapy comprised the process of care. The therapists employed at the centres during the time of data collection are presented in table $\mathbf{1}$.

From table 1 it becomes evident that three districts did not have occupational therapists deployed and therefore did not offer occupational therapy services. It also illustrates that the occupational therapists and physiotherapists are providing services to more than one centre in the same district.

\section{METHODS}

\section{Research Setting}

The study was conducted at all 39 CHCs which forms part of the Cape Town Metro District. The research setting was limited to these CHCs as the Comprehensive Service Plan for the provision of services in the Western Cape has package similar services for all the $\mathrm{CHCs}$ in this district (Provincial Government of The Western Cape, 2007). Patients accessing these centres should therefore receive equitable services. The $\mathrm{CHCs}$ in this district are located in urban and peri-urban geographical areas. The majority of people residing in these areas are classified as being part of the low to middle income groups (Stats SA, 2006).

\section{Study Design}

A cross sectional survey of all the therapists employed at the CHCs was conducted to collect data relating to the structure of rehabilitation. Process data relating to the services received was collected using a longitudinal design, while a cross sectional design was used to collect data relating to the number of therapy sessions received and the intensity of physiotherapy.

\section{Participants}

The population from which the information relating to the structure of rehabilitation was collected consisted of all therapists deployed at $\mathrm{CHCs}$ providing stroke rehabilitation in the Cape Metro District of the Western Cape. At the time of data collection a total of 6 occupational therapists and 16 physiotherapists provided services at 20 of the 39 CHCs comprising the research setting. The sample used to collect the process data consisted of 100 first ever

Table.1 Distribution of therapy services per district.

\begin{tabular}{|c|c|c|c|c|c|c|c|c|c|}
\hline & 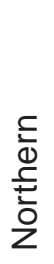 & 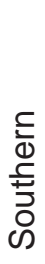 & 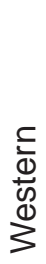 & $\begin{array}{l}\frac{0}{0} \\
\frac{0}{2} \\
\frac{0}{0} \\
\stackrel{2}{2}\end{array}$ & 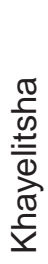 & 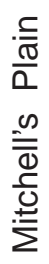 & 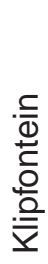 & 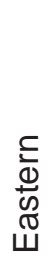 & 宽 \\
\hline No of $\mathrm{CHCs}$ & 4 & 5 & 5 & 7 & 4 & 4 & 5 & 5 & 39 \\
\hline $\begin{array}{l}\text { No of occupational } \\
\text { therapists }\end{array}$ & 1 & 1 & 0 & 2 & 0 & 0 & 1 & 1 & 6 \\
\hline No of physiotherapists & 1 & 2 & 2 & 2 & 2 & 1 & 4 & 2 & 16 \\
\hline $\begin{array}{l}\text { No of centres with } \\
\text { physiotherapist services }\end{array}$ & 1 & 3 & 2 & 3 & 3 & 1 & 4 & 3 & 20 \\
\hline $\begin{array}{l}\text { No of centres with } \\
\text { occupational therapist } \\
\text { services }\end{array}$ & 4 & 3 & 0 & 4 & 0 & 0 & 3 & 2 & 16 \\
\hline
\end{tabular}

stroke patients consecutively admitted to the $20 \mathrm{CHCs}$ between 1st June 2005 and 31st November 2007.

\section{Instrumentation}

The researcher developed a questionnaire based on a taxonomy conceptualized by (Hoening, Sloane, Horner, Zolkewitz, Duncan \& Hamilton, 2000), to assess structure and process of rehabilitation. The questionnaire requested information relating to the services and equipment available at the centres, as well as the occurrence of team meetings to discuss the management of patients. Three reviewers with extensive experience in the field of neurological rehabilitation provided feedback about the content validity of the questionnaire. The basis for their collective expertise included, but was not limited to, clinical practice, research and publication, teaching, as well as community-based intervention in neurological rehabilitation. After the necessary editorial changes the reviewers concurred that the questionnaire was valid in terms of its content. In addition, the questionnaire was sent to five therapists who did not form part of the sampling frame for completion. They verified the administration, logical flow, clarity of items and face validity of the instrument. Their feedback was that the questionnaire dealt with the proposed aims and objectives of the survey and was easy to complete as a self-report measure. 
To collect information relating to the process of rehabilitation in this sample a structured interview questionnaire and archived records were used. This questionnaire was developed by Putman (2006) for use in a study which investigated stroke rehabilitation across Europe known as the CERISE study (Putman, 2006). This questionnaire was named the "use of care" questionnaire in both the resent study and the CERISE Study and was completed at 2 and 6 months post stroke was used to collect information relating to the services received by the participants. Patient registers and therapists' records were used to extract data relating to the intensity and duration of therapy. This included; the date of admission to the centre, date of discharge or last date seen and the number of physiotherapy, occupational therapy and speech therapy sessions as well as the duration of physiotherapy treatment sessions.

\section{Procedure}

The questionnaires $(n=22)$ were hand delivered or posted to the therapists at the different centres together with a consent form and information sheet, as well as a self-addressed stamped envelop for the return of the questionnaire. The researcher followed-up non-respondents telephonically after three weeks to encourage participation and to increase the overall response rate to the survey. A researcher assistant called the centres thrice a week in order to ensure that stroke patients meeting the inclusion were recruited consecutively into the study. To complete the use of care questionnaire the researcher visited the patients at their homes when they were 2 and 6 months post stroke. To collect data relating to the frequency of physiotherapy, occupational and speech therapy and the number of hours of physiotherapy, the researcher made an appointment with the therapists and viewed the participants' records and the therapists' documents at a time that was convenient for the therapists. In cases where the admission and discharge dates for physiotherapy and occupational therapy differed, the earliest date of admission and latest date of discharge was recorded. This information was collected after the 6 month post stroke assessment period.
Table 2: Services and equipment available at the $20 \mathrm{CHCs}$ in the Cape Metro District of the Western Cape offering rehabilitation services.

\begin{tabular}{|c|c|}
\hline Variable & $\begin{array}{l}\text { Centres with } \\
\text { services and equipment } \\
\text { Number }\end{array}$ \\
\hline Service providers & \\
\hline Physiotherapist & 20 \\
\hline Medical officer & 20 \\
\hline Nurse & 20 \\
\hline${ }^{*}$ Orthopaedic nurse & 20 \\
\hline Physiotherapy assistant & 1 \\
\hline Occupational therapist & 10 \\
\hline Occupational therapy assistant & 5 \\
\hline Rehabilitation worker & 1 \\
\hline Home-based carer & 14 \\
\hline $\begin{array}{l}\text { Students (physiotherapy } \\
\text { (occupational therapy, speech therapy) }\end{array}$ & 10 \\
\hline Speech therapist & 2 \\
\hline Psychologist & 8 \\
\hline Dietician & 16 \\
\hline Social worker & 15 \\
\hline $\begin{array}{l}\text { Other: } \\
\text { (Health promoter and nutritional advisor) }\end{array}$ & 10 \\
\hline $\begin{array}{l}\text { Equipment } \\
\text { Hot packs }\end{array}$ & 20 \\
\hline Bobath couch & 19 \\
\hline Assistive devices & 19 \\
\hline Ultrasound & 19 \\
\hline Wheelchairs & 19 \\
\hline Ice & 17 \\
\hline Weights & 17 \\
\hline Gymballs & 16 \\
\hline Therapy mats & 16 \\
\hline Parallel bars & 15 \\
\hline Mirrors & 15 \\
\hline Interferential therapy & 13 \\
\hline Stairs & 9 \\
\hline Tens & 9 \\
\hline Suspension therapy & 8 \\
\hline Wall bars & 6 \\
\hline Nebuliser & 6 \\
\hline Suction machine & 5 \\
\hline Short Wave diathermy & 5 \\
\hline Wax & 4 \\
\hline Orthoses & 3 \\
\hline Functional electrical stimulation & 2 \\
\hline Others & 1 \\
\hline
\end{tabular}

${ }^{*}=$ Orthopaedic nurse = nurses who have been trained to follow-up persons with disabilities needs, e.g. wheelchair repairs 


\section{Data analysis}

The data was captured and analysed using Microsoft Excel and SPSS version 15. Data relating to the structure and process of rehabilitation was summarised using descriptive statistics and presented as frequencies and percentages.

\section{Ethical Considerations}

Ethical approval to conduct the study was granted by the University of the Western Cape. Permission to conduct the study was also requested from the medical superintendent of the Community Health Services Organisation. Written informed consent was obtained from all participants or their families where the participants could not provide consent due to communication problems or cognitive limitation. The patients were assured of confidentiality and anonymity as no participant names were recorded during the capturing and analysis of the data. The participants were also ensured of their right to withdraw from the study at any stage.

\section{RESULTS}

The results that will be presented include the availability of staff and equipment at the $\mathrm{CHCs}$, the services received by the participants, the number of therapy sessions and the intensity of physiotherapy provided. Twenty therapists provided data relating to the structure of rehabilitation. Data relating to the process of care was collected from 100 stroke patients. Twelve patients dropped out of the study between baseline and 2 months therefore only 88 patients completed questionnaires at this assessment period. A further 12 dropped out between 2 and 6 month assessment periods. Therefore data collected at the 6 months assessment period was only collected from 76 patients.

\section{Availability of Staff and Equipment at the $\mathrm{CHCs}$}

The number and percentage of staff and equipment available at the centres providing rehabilitation services as reported by the therapist when completing the questionnaires are presented in Table 2.

All the centres had physiotherapy services either on a part-time or fulltime basis $(n=20)$, medical services $(n=20)$ and orthopaedic nursing services $(n=20)$ available. With regards to auxiliary rehabilitation services more occupational therapy $(n=10)$ than physiotherapy assistants $(n=1)$ were employed at this level with only one of the centres having the services of staff formally employed as rehabilitation workers. Fourteen centres offered home-based care.

The majority of the centres (19) had a Bobath plinth and assistive devices which included walking aids such as walking sticks, crutches and quadrupods. Gymballs, therapy mats, parallel bars and mirrors were not available in respectively 4 and 5 percent of the centres. Only two $(9.1 \%)$ of the therapists indicated having team meetings to discuss the rehabilitation of stroke patients.

\section{Services received by the participants}

Table 3 presents the information the participants provided regarding the services they received.

The table 3 shows that the majority of the participants were treated by physiotherapists at both the 2 and 6 month assessment periods. Twenty percent more participants were receiving medical services and seven percent more social work services in the four months prior to the six months follow-up than during the first two months post stroke onset.

\section{Number of therapy treatment sessions}

Table 4 summarises the number and percentage of physiotherapy, occupational therapy and speech therapy sessions speech, the participants received.

Table 4 illustrates that more than two thirds of the participants received between 1 and 5 physiotherapy, occupational therapy and speech therapy sessions. Sixty eight percent of the participants

Table 3: Services received by the participants as reported at 2 and 6 months post stroke.

\begin{tabular}{|c|c|c|}
\hline \multirow[t]{2}{*}{ Services Received } & \multicolumn{2}{|c|}{ Number (\%) of Participants } \\
\hline & $\begin{array}{l}2 \text { month } \\
\mathrm{n}=88(\%)\end{array}$ & $\begin{array}{l}6 \text { month } \\
\mathrm{n}=76(\%)\end{array}$ \\
\hline Physiotherapy & $87(98.8)$ & $44(57.9)$ \\
\hline Medical care & $55(62.5)$ & $63(82.9)$ \\
\hline Occupational therapy & $22(25.0)$ & $16(21.1)$ \\
\hline Speech therapy & $8(9.1)$ & $6(7.9)$ \\
\hline Home-based care & $4(4.5)$ & $1(1.3)$ \\
\hline Nursing care & $4(4.5)$ & $3(3.9)$ \\
\hline Other & $3(3.4)$ & $6(7.9)$ \\
\hline Social worker & $2(2.3)$ & $7(9.2)$ \\
\hline Intervention by dietician & $2(2.3)$ & $2(2.6)$ \\
\hline Rehabilitation worker & $1(1.1)$ & $1(1.3)$ \\
\hline Specialist services & $1(1.1)$ & $2(2.6)$ \\
\hline
\end{tabular}

Table 4: Number of physiotherapy, occupational therapy and speech therapy sessions the participants received between baseline and six months post stroke $(\mathrm{N}=100)$.

\begin{tabular}{|l|l|l|l|}
\hline $\begin{array}{l}\text { Number of therapy } \\
\text { sessions }\end{array}$ & \multicolumn{3}{|c|}{ Number (\%) of participants } \\
\hline 0 & PT & OT & Speech \\
$65(65.0)$ & $92(92.0)$ \\
\hline $1-5$ & $3(3.0)$ & $22(22.0)$ & $8(8.0)$ \\
\hline $6-10$ & $68(68.0)$ & $5(5.0)$ & $0(0)$ \\
\hline $11-15$ & $15(15.0)$ & $4(4.0)$ & $0(0)$ \\
\hline $16-36$ & $6(6.0)$ & $4(0)$ & $0(0)$ \\
\hline
\end{tabular}

Key: PT=Physiotherapy, OT=Occupational Therapy, Speech=Speech Therapy. 
received between 1 and 5 physiotherapy sessions, while $22 \%$ percent of the participants received between one and five occupational therapy sessions. Only 8\% of the patients received speech therapy.

\section{Intensity of Physiotherapy treatment}

Only data relating to the intensity of physiotherapy was available in the records. The intensity of physiotherapy is expressed as the total treatment time the patients received. Physiotherapists record the treatment session in units of 5 minutes. The total treatment time received by each participant was calculated by adding the number of units and multiplying by 5 . This total was then converted to hours. The total treatment time received by the participants is presented in table $\mathbf{5}$.

The median (Q1-Q3) number of hours was 1.83 (0.83- 3.9) hours. Almost half of the participants (49\%) received between 1 and 4 hours of physiotherapy.

\section{DISCUSSION}

\section{Staff and services available for rehabilitation}

The aim of the present study was to determine the structure and process of rehabilitation at Community Health Centres in the Western Cape. When considering all the $\mathrm{CHCs}$ in the district the results revealed that there is a lack of therapy staff employed at CHCs in the Metropole Region of the Western Cape Province. Of the $39 \mathrm{CHCs}$ situated in various districts only 20 offered rehabilitation services. Although all centres offering rehabilitation provide physiotherapy only half offered occupational therapy services and only 2 offered speech therapy services which were provided by students. The limited availability of therapy services is in contrast to what is recommended by various policy documents. The National Rehabilitation policy of South Africa advocates that rehabilitation services provided should be "...equitable, affordable and accessible to all..." (Department of Health, 2000, p:8). South Africa has also adopted the Primary Health Care approach to provide healthcare services to its population. In this approach healthcare services that should be provided include preventative, promotive, curative as well as rehabilitative services. The lack of rehabilitation services meant that a large percentage of the population residing in this geographical area did not have access to rehabilitation services at a primary level of care which should be inclusive of a primary health care approach. It also meant that clients have to travel long distances to receive rehabilitation. The lack of services is also a challenge to therapists who need to refer patients from other levels of care e.g. tertiary and secondary level. Patients can receive intensive in-patient rehabilitation and not be followed-up in the community which might facilitate their re-integration into their homes and communities. Therapists working at $\mathrm{CHCs}$ often conduct home visits to determine the re-integration of patients into their homes. The consistent report from staff surveyed was that even though rehabilitation services were available, the services at $\mathrm{CHCs}$ are not optimally coordinated. Only 2 of the therapists in the present study reported that they had regular meetings to discuss the progress of stroke patients. It is important to note that there is empirical evidence suggesting that it is the coordination of services, such as physiotherapy, occupational

Table 5: Physiotherapy treatment time $(\mathrm{N}=100)$.

\begin{tabular}{|l|l|l|}
\hline Time (hours) & Number of participants & Percentage \\
\hline 0 & 3 & 3.0 \\
\hline$<1$ & 24 & 24.0 \\
\hline $1-2$ & 25 & 25.0 \\
\hline$>2-4$ & 24 & 24.0 \\
\hline$>4-7$ & 10 & 10.0 \\
\hline$>7-10$ & 8 & 8.0 \\
\hline$>10$ & 6 & 6.0 \\
\hline
\end{tabular}

therapy, speech therapy, nursing and medical services, that results in improved outcome, and not only the availability of services (Langhorne \& Pollock, 2002). Similarly, Young and Forster (2007) reported that coordinated stroke care is beneficial for stroke patients irrespective of the level of severity, age or gender of the patients. One of the challenges to optimally coordinate services is that stroke is but one of the many conditions treated by the health professionals at the CHCs. Thus it becomes evident that the therapeutic value of rehabilitation is curtailed by the lack of optimal coordination at $\mathrm{CHCs}$ in the Western Cape.

Information related to the structure of rehabilitation gathered during the survey of therapists employed at the CHCs revealed that even though the majority of the centres (75\%) had equipment needed for the rehabilitation of stroke patients the equipment was not equally available at all the centres. This however would not impact negatively on the rehabilitation of stroke patients, as no evidence is available indicating that the use of specific equipment would result in better outcomes of stroke patients.

\section{Availability and Intensity of Services Received}

The results of the study revealed that a wide range of services which include medical services, social work, dietician, physiotherapy, and occupational therapy are available to stroke patients at certain CHCs. This is disconcerting as not many of the patients reported receiving the above mentioned services. The majority of the participants consulted a medical officer. Similar to what is found in the literature, physiotherapy was the therapeutic service most frequently received by the participants (Jiménez et al., 2000; Whitelaw et al., 1993). At the 6 month follow-up assessment period, physiotherapy, followed by occupational therapy, and then speech therapy were the rehabilitation services most frequently received by the participants. These results are similar to those found in a follow-up study conducted in Cape Town by De Villiers et al., (2006) where more of the participants $(27 \%)$ received physiotherapy than occupational therapy (5\%) after being discharged from hospital. 
In addition to the many participants not receiving optimal rehabilitation services the intensity and frequency of therapy is much less when compared to what is found in other out-patient community-based settings in developed countries (Young and Forster, 1992). In the present study the participants received physiotherapy sessions which averaged less than half an hour. This is much less than what is documented in other studies of patients receiving day hospital rehabilitation where patients receive up to one hour of rehabilitation per session (Hershkovitz et al., 2004). The number of treatment sessions in total was also less than what was provided in a study conducted in the United Kingdom (UK) by Young \& Forster, (1992). Stroke patients treated in a day hospital in the Bradford Community Trial in the UK attended for a median of 31 times in a 6 month period (Young \& Forster, 1992).

The decreased number of therapy sessions could be linked to availability of therapy staff or therapists workload. It should however be noted that an increase in the number of therapists do not also always result in an increase in the intensity of therapy. De Wit et al., (2005) suggest that an increase in therapy intensity is dependent on the structure of the rehabilitation sessions and not necessarily of the number of staff available. It should also be noted that the decreased frequency of therapy received by participants in the study could also be as a result of patients not attending follow-up sessions. An earlier study conducted at one of the tertiary hospitals in the Western Cape revealed that stroke patients could not attend follow-up treatment sessions due to a lack of transport to access the centres (Whitlaw et al., 1993). The present study however did not explore accessibility of the centres.

\section{CONCLUSION AND RECOMMENDATIONS}

According to the Comprehensive Service Plan of the Western Cape CHCs, which are identified as low intensity rehabilitation facilities should offer rehabilitation services which include physiotherapy and occupational therapy. Rehabilitation is also part of the package of services that should be provided as part of the comprehensive services that should be included in a primary health care approach. One can therefore conclude rehabilitation services should be available to patients accessing CHCs. The results of the present study however revealed that there is a lack of therapy services to provide rehabilitation to stroke patients at the $\mathrm{CHCs}$ in the Western Cape. Services that are currently available are not coordinated which could negatively impact effective rehabilitation. Findings about the process of rehabilitation indicated that the frequency of physiotherapy, occupational therapy and speech therapy as well as the number of hours of physiotherapy was low. It should however be noted that the decreased intensity of therapy received by stroke patients could either be as a result of lack of services as well as the inability to access the centres. In addition to advocating for an increase in physiotherapy, occupational therapy and speech therapy services at $\mathrm{CHCs}$ in the Western, the researcher would also like to recommend that further research is done to determine if the lack of intensity is also not linked to a lack of accessibility of the centres.

\section{REFERENCES}

Department of Health. 2000 Rehabilitation For All. National Rehabilitation Policy.

De Villiers, L., Kalula, S., Bryer, A., \& Ferreira, M. 2006 Stroke Outcomes in Sub-Saharan Africa. International Journal Of Rehabilitation 11(1), 25

De Wit, L., Putman, K., Lincoln, N., Baert, I., Berman, P., Beyens, H., et al. 2006 Stroke Rehabilitation in Europe. What Do Physiotherapists and Occupational Therapists Actually Do? Stroke, 37, 1483 - 1489.

De Wit, L., Putman, K., Dejaeger, E., Baert, I., Berman, P., Bogaerts, K., et al. 2005 Use of Time by Stroke Patients A Comparison of Four European Rehabilitation Centres. Stroke, 36, 1977-1983.

Fritz, v. 1995 Stroke, including Rehabilitation. Chronic Diseases of Lifestyle in South Africa. MRC Technical Report, chapter X 161-175

Hale, L., \& Wallner, P. 1996 The Challenge of Service Provision in South Africa for Patients with hemiplegia. Physiotherapy, 62(3), 156-158.

Helander, E. 1999 Prejudice and Dignity An Introduction to Community-Based Rehabilitation (Second ed.). New York: United Nations Development Programme.
Hershkovitz, A., Beloosesky, Y., Brill, S., \& Gottlieb, D. 2004) Is a day hospital rehabilitation programme associated with reduction of handicap in stroke patients? Clinical Rehabilitation, 18, 261 - 266.

Hoenig, H., Horner, R., Duncan, P., Clipp, E., \& Hamilton, B. 1999 New Horizons in stroke rehabilitation research. Journal of Rehabilitation Research and Development, 36(1), 19-31.

Hoenig, H., Sloane, R., Horner, R., Zolkewitz, M., Duncan, P., \& Hamilton, B. 2000 A Taxonomy for Classification of Stroke Rehabilitation Services. Archives of Physical Medicine and Rehabilitation, 81, 853-862.

Jiménez, M., de Pedro-Cuesta, J., Almazan, J., \& Widen Holmqvist, L. 2000 Stroke Patients in South Madrid: Function and Motor Recovery, Resource Utilization, and Family Support. Stroke, 31, 1352 - 1359.

Langhorne, P., \& Pollock, A. 2002 What are the components of effective stroke unit care? Age and Ageing, 31, 365 - 371.

Provincial Government of the Western Cape. 2007 Comprehensive Service Plan for the Implementation of 2010.

Putman, K. 2006 Collaborative Evaluation of Rehabilitation in Stroke Across Europe. Unpublished Thesis. Department of Health Sciences and Medical Sociology, Faculty of Medicine and Pharmacy, Vrije Universiteit Brussel.

Rhoda, A., \& Hendry J. 2006 Rehabilitation of Stroke Patients treated at a Community Based Rehabilitation Centre. Journal of Community and Health Sciences, 1(1)47-53

Rhoda, A. 2002 A profile of stroke patients treated at the Bishop Lavis Rehabilitation Centre: 1995-1999. Unpublished Thesis Stellenbosch University, Cape Town.

South African Medical AssociationNeurological Association of South Africa working group 200 Stroke Therapy guideline. South African Medical Journal, 90(3), 274-306

Statistics South Africa 2006 Provincial Profile 2004: Western Cape. Pretoria, Statistics South Africa.

Stroke Unit Trialists' Collaboration. 2006 Organised inpatient (stroke unit) care for stroke. Cochrane Database of Systematic Reviews. Issue 4 Art. No.: CD000197. DOI:10.1002/14651858.CD0000197.pub2

Whitlaw, D., Meyer, C., Bawa, S., \& Jennings, K. 1993 Post-discharge follow-up of stroke patients at Groote Schuur hospitala prospective study. South African Medical Journal, 84(2), 11-13.

World Health Organisation. 2001 International Classification of Functioning, Disability and Health: ICF short version: WHO Library Cataloguing-in-Publication Data.

Young, J., \& Forster, A. 1992 The Bradford community stroke trial: Results at six months. British Medical Journal, 304, 1085-1089.

Young, J., \& Foster, A. 2007 Review of stroke rehabilitation. British Medical Journal, 334(7584), 86 - 90. 